OPEN ACCESS

Edited by:

Pasquale Pisapia,

University of Naples Federico II, Italy

Reviewed by:

Ilaria Attili,

European Institute of Oncology (IEO),

Valerio Gristin

University of Palermo, Italy

${ }^{*}$ Correspondence:

Jun Wang

ggjun2005@126.com

Specialty section:

This article was submitted to

Pharmacology of Anti-Cancer Drugs,

a section of the journal

Frontiers in Oncology

Received: 22 January 2022

Accepted: 14 February 2022

Published: 04 March 2022

Citation:

Ma L, Xiao J, Guan Y, Wu D, Gu T and Wang J (2022) SDK1-ALK Fusion in a

Lung Adenocarcinoma Patient

With Excellent Response to ALK Inhibitor Treatment: A Case Report.

Front. Oncol. 12:860060.

doi: 10.3389/fonc.2022.860060

\section{SDK1-ALK Fusion in a Lung Adenocarcinoma Patient With Excellent Response to ALK Inhibitor Treatment: A Case Report}

\author{
Lin $\mathrm{Ma}^{1,2,3}$, Junjuan Xiao ${ }^{1,2,3}$, Yaping Guan ${ }^{1,2,3}$, Dongfang $W^{4}$, \\ Tiantian $\mathrm{Gu}^{4}$ and Jun Wang ${ }^{1,2,3 *}$ \\ ${ }^{1}$ Department of Oncology, The First Affiliated Hospital of Shandong First Medical University \& Shandong Provincial \\ Qianfoshan Hospital, Jinan, China, ${ }^{2}$ Shandong Lung Cancer Institute, Jinan, China, ${ }^{3}$ Shandong Key Laboratory of \\ Rheumatic Disease and Translational Medicine, Jinan, China, ${ }^{4}$ YuceBio Technology Co., Ltd, Shenzhen, China
}

Background: Rearrangements of Anaplastic lymphoma kinase (ALK) have been discovered as a novel driver mutation in patients with non-small-cell lung cancer (NSCLC). Patients' responses to ALK tyrosine kinase inhibitors (TKIs) may vary depending on the variations of $A L K$ rearrangements they have. It is imperative for clinicians to identify druggable $A L K$ fusions in routine practice.

Case Presentation: In this study, we discovered a rare ALK rearrangement type (SDK1$A L K)$ in a Chinese lung adenocarcinoma patient who responded well to ALK inhibitor SAF189s. The positive expression of ALK in lung biopsy tissue was verified by $\mathrm{HC}$ analysis. $A$ new SDK1-ALK fusion was discovered using NGS. The patient was treated with SAF189s (160 mg per day) as a first-line therapy and went into continuous remission, with a 12 months progression-free survival at the last follow-up.

Conclusion: This is the first case of SDK1-ALK fusion with an excellent response to an ALK inhibitor, which will provide better understanding of ALK-TKI applications for NSCLC patients with $A L K$ fusion in the future.

Keywords: non-small-cell lung cancer, ALK rearrangement, ALK inhibitor, SAF-189s, SDK1-ALK

\section{INTRODUCTION}

Anaplastic lymphoma kinase $(A L K)$ rearrangements account for approximately $5 \%$ of patients with non-small-cell lung cancer (NSCLC) and represent as a critical therapeutic target in clinical practice (1). Patients with ALK rearrangements in NSCLC are usually younger and light smokers. ALK tyrosine kinase inhibitors (TKIs) provide a marked objective response rate (ORR) and impressive clinical benefit for patients with $A L K$-rearranged lung cancer. The first-generation ALK inhibitor was crizotinib, developed after the discovery of chromosomal rearrangement involving the ALK and echinoderm microtubule-associated protein like 4 (EML4) genes in NSCLC in 2007. Secondgeneration inhibitors including ceritinib, alectinib, and brigatinib, were authorized for treatment in ALK-positive patients after then. As third-generation inhibitors, lorlatinib and ensartinib have been developed for the treatment of NSCLC patients who have acquired resistance to prior ALK inhibitor 
treatment (2). In 2021, the FDA has expanded the approval for lorlatinib to include an new indication for the first-line treatment of patients with ALK-positive NSCLC (3). In our case, SAF-189s is a new ALK inhibitor that has the ability to overcome various resistance mutations.

Numerous ALK fusion partner genes, such as EML4 (94\%), KIF5B (1.6\%), and other variants (4.7\%), have been identified with the rapid development and application of next-generation sequencing (NGS) (4). For patients who are using ALK inhibitors, distinct fusion patterns are linked to varying clinical outcomes (5). It is imperative for clinicians to delineate a "response diagram" of patients with unknown $A L K$ fusion variants treated with ALK inhibitors. Here, we describe an undocumented ALK rearrangement (SDK1-ALK fusion) in a lung adenocarcinoma patient who exhibited a remarkable response to SAF-189s.

\section{CASE PRESENTATION}

In December 2020, a 47-year-old man with a history of smoking came to our hospital with a paroxysmal dry cough that had lasted about a year and left subcostal pain for two months, which aggravated after deep inhalation. A computed tomography (CT) scan revealed a mass in the left lower lobe measuring $8.1 \times 6.1$ $\mathrm{cm}$, multiple enlarged lymph nodes in left hilum and mediastinum, obstructive pneumonia in the left lung, and left pleural effusion. Radionuclide bone scan and magnetic resonance imaging revealed no evidence of bone or brain metastasis. His medical history was not remarkable. The pathology of this patient's lung cancer was verified through a CT-guided percutaneous fine-needle lung biopsy. According to the American Joint Committee on Cancer Staging Manual, 8th edition, he was finally diagnosed with stage IV lung adenocarcinoma (cT4N2M1). The biopsy specimen was subjected to next-generation sequencing (NGS), which revealed a hitherto unknown SDK1 Exon36-ALK Exon20 fusion variant (abundance: 9.2\%) (Figure 1A). Further immunohistochemical (IHC) analysis indicated the positive expression of ALK protein (clone D5F3, Ventana) (Figure 1B).

It is well known that ALK inhibitors, such as Crizotinib and alectinib, are pricey. If the patient had maintained his treatment, he would have been in serious financial trouble. SAF-189s is a new selective ALK inhibitor, and the patient satisfied all of the criteria for clinical trial inclusion. He provided informed permission. On January 13, 2021, the patient was then enrolled onto a phase I/II clinical trial evaluating SAF-189s in NSCLC (Clinical Trials.gov number, NCT00585195) after a thorough enrollment assessment and began with oral administration of SAF-189s at a dose of $160 \mathrm{mg}$ per day. After 10 days of medication, his symptoms considerably improved. On February, 2021, done 2 months after treatment initiation, a chest CT scan revealed a partial response (42 percent reduction in the sum of the target lesion's longest diameters per RECIST 1.1) (Figure 1C), and further radiological assessments confirmed his continuing tumor response to this
ALK inhibitor. At the time of the latest follow-up, he tolerated well with only grade 1 rash and had a 12-month progression-free survival (Figure 1D). Now he continues to be in partial response and keeps on SAF-189s treatment.

\section{DISCUSSION}

ALK fusions are more common in patient with younger age and light or never smoking history. Like epidermal growth factor receptor (EGFR) mutations that were frequently found in female and adenocarcinoma patients, ALK gene aberrations are routinely found in patients with adenocarcinoma histological subtype. Remarkably, brain metastases are more likely to be found in this subset of patients (6). Many researchers focused on the heterogeneous response mechanism of EGFR-mutated NSCLC patients during EGFR-TKI treatment as the significant proportion of EGFR-positive cases in NSCLC. In patients with $A L K$-rearranged lung cancer, little is known regarding the effectiveness and relevant mechanism of ALK-TKIs. Firstly, different $A L K$ fusion partners resulted in different levels of ALK expression and protein stabilities, which may be interpreted as different ALK-TKI sensitivities in individuals (7, 8). Secondly, the signaling networks activated by ALK are intricate due to the various alterations found in individuals. Phosphorylated ERK and STAT3 levels were found to be upregulated in EML4-ALK-positive cell lines. Delineation of the extensive ALK signaling network is critical for the development of ALK inhibitor-based combination therapies.

A number of biomarkers should be tested on account of the incredible accomplishment of precision medicine improving NSCLC patients' outcomes significantly. Patients with advanced-stage NSCLC always have a limited amount of tissue for molecular analysis. NGS is a valid approach able to detect sufficient gene alterations simultaneously, and it can be started with nucleic acids recovered from patients' cancer tissues in situ or liquid biopsy samples. According to the KWAY Italian multicenter cost evaluation research, the implementation of NGS saves personnel time spent to testing activities and lowers the overall cost of testing per patient $(9,10)$. Fluorescence in situ hybridization and IHC are gold standards for $A L K$ mutation testing. NGS further identify the complex $A L K$ rearrangement in NSCLC, which can be an effective complement in clinical decision-making and an efficient approach to find novel variants and fusion partners (11).

In this case, NGS was used for the identification of an uncommon $S D K 1-A L K$ fusion in a patient with lung adenocarcinoma for the first time. SDK, encoded by the Sidekick gene was initially discovered in Drosophila, which is one of the largest members in immunoglobulin superfamily (12). $S d k 1$ and $S d k 2$ are vertebrate ortholog of $S d k$. Genome-wide studies revealed that $S D K 1$ polymorphism is related to neurological disorders $(13,14)$. Reports clarified that the SDK gene is extremely fragile, and that $S D K$ mutations can be found in a variety of human cancers $(15,16)$. Ren et al. found that SDK1-AMACR fusion might be a crucial factor in progression of 
A
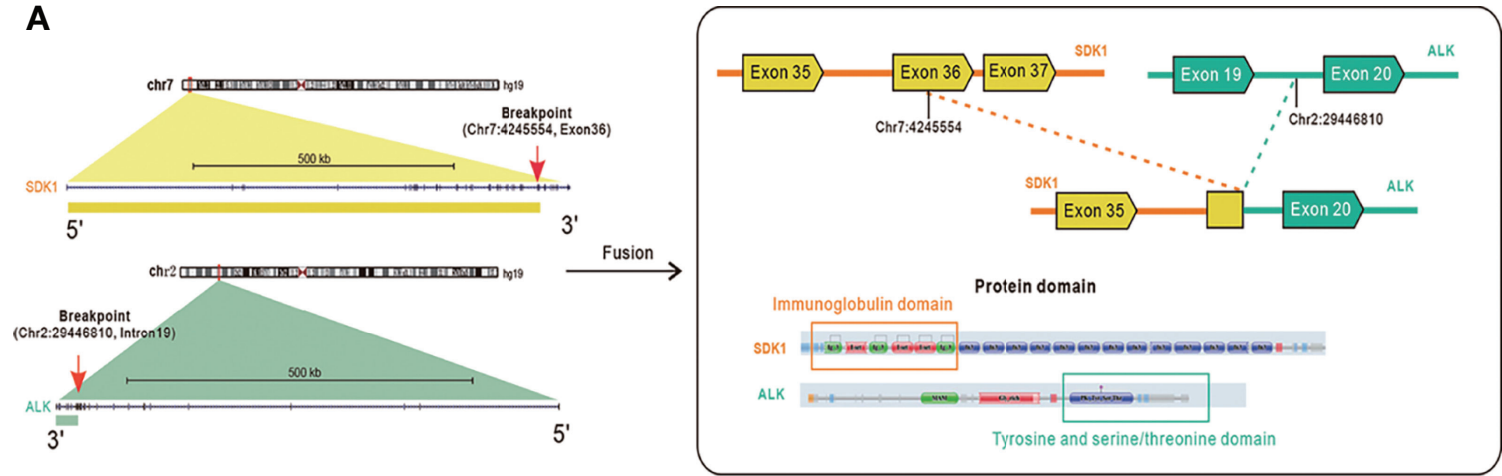

B

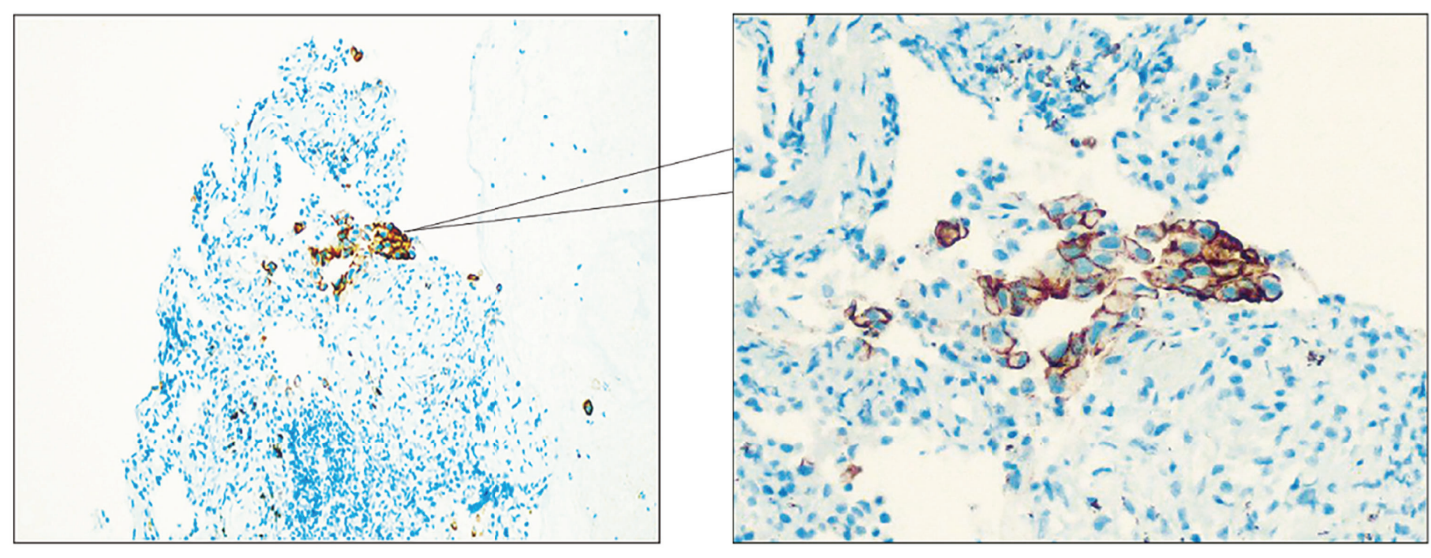

C

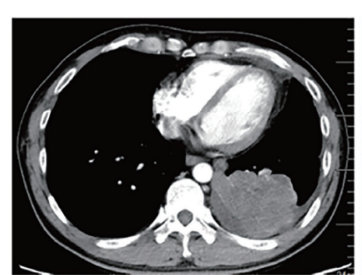

before treatment

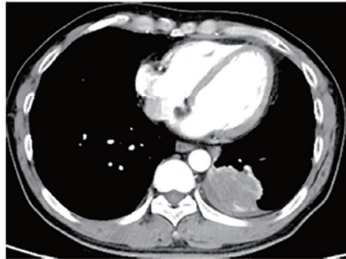

after 1 month treatment

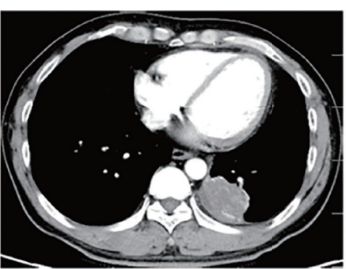

after 2 month treatment

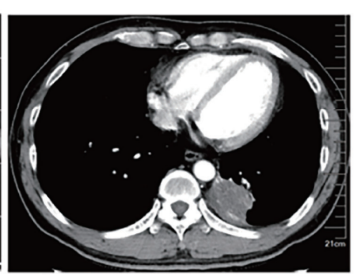

after 4 month treatment

D

\begin{tabular}{|c|c|c|c|c|}
\hline 2020.12 .24 & 2020.12 .26 & 2021.01 .13 & 2021.02 & $\begin{array}{l}\text { The latest } \\
\text { follow-up }\end{array}$ \\
\hline Diagnosis & $\begin{array}{c}\text { NGS } \\
\text { detection }\end{array}$ & $\begin{array}{c}\text { Administration } \\
\text { of SAF-189s }\end{array}$ & $\begin{array}{l}\text { CT scan of the } \\
\text { first follow-up }\end{array}$ & PFS:12 months \\
\hline
\end{tabular}

FIGURE 1 | A Chinese patient with adenocarcinoma harboring a novel SDK1-ALK fusion variant exhibited excellent response to ALK inhibition. (A) The schematic diagram. (B) $I H C$ showed the positive expression of ALK protein. Cancer cells showed an uneven stippled cytoplasmic and perimembranous staining pattern.

(C) Compared with the baseline images, the size of the lesion in the left lower lobe, as well as lymph nodes in left hilum and mediastinum was significantly reduced after this patient was treated with SAF-189s for 1 month, 2 months, and 4 months. (D) The timeline diagram of this case. At the time of the latest follow-up, he tolerated well with only grade 1 rash and had a 12-month progression-free survival. Now he continues to be in partial response and keeps on SAF-189s treatment. 
prostate cancer (17). The specific function of SDK1 in NSCLC still needs further study. The homophilic binding of Sdk1 ectodomain regions is required for cell-cell aggregation (18). Mutations in SDK1 gene have been found in the lung tissue of asbestos-exposed patients and cancer specimens of stage I lung adenocarcinoma, which may be responsible for dysfunction of cell adhesion in tumor progression $(19,20)$. In our case, the SDK1 Exon36-ALK Exon20 fusion protein contained Sdk1 ectodomain regions and the ALK kinase domain, which might have resulted in ligand-independent dimerization and hence continuous activation of ALK.

Targeted therapy has brought notable clinical benefits to ALK positive NSCLC patients. Crizotinib has become the standard first-line oral TKI therapy in patients with ALK-positive metastatic NSCLC based on the outcomes of the PROFILE 1007 trial and the phase III PROFILE 1014 clinical study. The second-generation ALK TKIs ceritinib and alectinib obtained expedited approval as first-line medications based on the clinical trials ASCEND-4 and ALEX. Another second-generation TKI brigatinib exhibited a 71\% ORR in the phase 3 ALTA- 1L trial, making it the new first-line medication for advanced-stage NSCLC patients. Lorlatinib, a third-generation ALK TKI, achieved a $72 \%$ improvement in PFS compared with crizotinib as a first-line therapy in the phase III CROWN trial $(3,21)$. Secondary mutations in the ALK tyrosine kinase domain, such as L1196M, C1156Y, G1202R, and G1269A, are the most common cause of ALK-targeted therapeutic resistance, termed as ALKdependent resistance. ALK gene amplification and activation of alternative pathways, including epidermal growth factor receptor (EGFR), hepatocyte growth factor receptor, and insulin-like growth factor 1 receptor, represent other resistance mechanisms (2).

SAF-189s is a novel selective ALK inhibitor and can overcome multiple resistance mutation. In a multicenter I/II study, all enrolled 34 ALK-positive patients responded well to SAF-189s treatment, with a confirmed PR of $50 \%$ and an unconfirmed PR of $11.7 \%$. In 24 patients who progressed on previous first-line crizotinib or ceritinib treatment, a confirmed PR was $47.6 \%$ (22). In the nude mice xenograft model of SDC4-ROS1 fusion NSCLC, SAF-189s induced tumor regression and exhibited notable prolonged and durable efficacy and was more potent than crizotinib and comparable to lorlatinib against G2032R mutant-driven tumors (23). The present untreated lung cancer patient exhibited excellent response to SAF-189s treatment with manageable toxicities.

Immune checkpoint inhibitors, which can modulate tumoral immunosuppression and reactive host immunity, promising long-term disease control in a segment of patients with advanced NSCLC. As a result, increasing emphasis is being placed on the combination strategies of immunotherapy and targeted therapy. However, the present research findings on ALK TKIs in combination with immunotherapy are still ambiguous. The CheckMate 370 phase $1 / 2$ study and TATTON trial, for example, had halted their enrolments due to severe intolerant toxicities. Conversely, research of alectinib plus atezolizumab showed a manageable side-effects profile with excellent antitumor activity (ORR 85\%). The understanding of biological mechanisms underlying immune-targeted combinations still need further clinical investigations and NGS will be a valid tool in future decision-making (24).

\section{CONCLUSION}

In summary, this is the first case report involving SDK1-ALK fusion detected in a Chinese patient with advanced lung adenocarcinoma by using NGS-based cancer genomic DNA profiling in the clinic. In this case, the SDK1-ALK-rearranged lung cancer in this patient is susceptible to treatment with a new ALK inhibitor SAF-189s, which is now being studied in a clinical trial. Thus, our study provides a new druggable target for NSCLC driver mutation in routine practice.

\section{DATA AVAILABILITY STATEMENT}

The datasets for this article are not publicly available due to concerns regarding participant/patient anonymity. Requests to access the datasets should be directed to the corresponding author.

\section{ETHICS STATEMENT}

The studies involving human participants were reviewed and approved by Ethics Committee of The First Affiliated Hospital of Shandong First Medical University. The patients/participants provided their written informed consent to participate in this study. Written informed consent was obtained from the individual(s) for the publication of any potentially identifiable images or data included in this article.

\section{AUTHOR CONTRIBUTIONS}

LM: Data curation, Writing- Original draft preparation. JX: Writing - review \& editing. TG: Visualization, Investigation. DW: Software. JW: Supervision. All authors contributed to the article and approved the submitted version.

\section{FUNDING}

This study was supported by the National Natural Science Foundation of China (Grant No. 81572875), CSCO-MSD Cancer Research Foundation (Grant No. Y-MSD2020-0350), CSCO-PILOT Cancer Research Foundation (Grant No. Y2019AZMS-0440), Wu Jieping Medical Foundation for Clinical Scientific Research (Grant No. 320.6750.2020-12-16), and the Natural Science Foundation of Shandong Province (Grant No. ZR202102190539). 


\section{REFERENCES}

1. Ramalingam SS, Owonikoko TK, Khuri FR. Lung Cancer: New Biological Insights and Recent Therapeutic Advances. CA Cancer J Clin (2011) 61:91112. doi: $10.3322 /$ caac. 20102

2. Golding B, Luu A, Jones R, Viloria-Petit AM. The Function and Therapeutic Targeting of Anaplastic Lymphoma Kinase (ALK) in Non-Small Cell Lung Cancer (NSCLC). Mol Cancer (2018) 17(1):52. doi: 10.1186/s12943-0180810-4

3. Shaw AT, Bauer TM, de Marinis F, Felip E, Goto Y, Liu G, et al. CROWN Trial Investigators. First-Line Lorlatinib or Crizotinib in Advanced ALKPositive Lung Cancer. N Engl J Med (2020) 383(21):2018-29. doi: 10.1056/ NEJMoa2027187

4. Mitiushkina NV, Tiurin VI, Iyevleva AG, Kholmatov MM, Filippova EA, Moiseyenko FV, et al. Variability in Lung Cancer Response to ALK Inhibitors Cannot be Explained by the Diversity of ALK Fusion Variants. Biochimie (2018) 154:19-24. doi: 10.1016/j.biochi.2018.07.018

5. Camidge DR, Bang YJ, Kwak EL, Iafrate AJ, Varella-Garcia M, Fox SB, et al. Activity and Safety of Crizotinib in Patients With ALK-Positive Non-SmallCell Lung Cancer: Updated Results From a Phase 1 Study. Lancet Oncol (2012) 13:1011-9. doi: 10.1016/S1470-2045(12)70344-3

6. Rodig SJ, Mino-Kenudson M, Dacic S, Yeap BY, Shaw A, Barletta JA, et al. Unique Clinicopathologic Features Characterize ALK-Rearranged Lung Adenocarcinoma in the Western Population. Clin Cancer Res (2009) 15 (16):5216-23. doi: 10.1158/1078-0432.CCR-09-0802

7. Hallberg B, Palmer RH. Mechanistic Insight Into ALK Receptor Tyrosine Kinase in Human Cancer Biology. Nat Rev Cancer (2013) 13:685-700. doi: $10.1038 / \mathrm{nrc} 3580$

8. Heuckmann JM, Balke-Want H, Malchers F, Peifer M, Sos ML, Koker M, et al. Differential Protein Stability and ALK Inhibitor Sensitivity of EML4-ALK Fusion Variants. Clin Cancer Res (2012) 18:4682-90. doi: 10.1158/10780432.CCR-11-3260

9. Pisapia P, Pepe F, Baggi A, Barberis M, Galvano A, Gristina V, et al. Next Generation Diagnostic Algorithm in Non-Small Cell Lung Cancer Predictive Molecular Pathology: The KWAY Italian Multicenter Cost Evaluation Study. Crit Rev Oncol Hematol Actions (2022) 169:103525. doi: 10.1016/ j.critrevonc.2021.103525

10. Russo A, Incorvaia L, Del Re M, Malapelle U, Capoluongo E, Gristina V, et al. The Molecular Profiling of Solid Tumors by Liquid Biopsy: A Position Paper of the AIOM-SIAPEC-IAP-Sibioc-SIC-SIF Italian Scientific Societies. ESMO Open Actions (2021) 6(3):100164. doi: 10.1016/j.esmoop.2021.100164

11. Peled N, Palmer G, Hirsch FR, Wynes MW, Ilouze M, Varella-Garcia M, et al. Next-Generation Sequencing Identifies and Immunohistochemistry Confirms a Novel Crizotinib-Sensitive ALK Rearrangement in a Patient With Metastatic Non-Small-Cell Lung Cancer. J Thorac Oncol (2012) 7:e14-6. doi: 10.1097/ JTO.0b013e3182614ab5

12. Amacher JF, Brooks L, Hampton TH, Madden DR. Specificity in PDZ-Peptide Interaction Networks: Computational Analysis and Review. J Struct Biol (2020) 4:100022. doi: 10.1016/j.yjsbx.2020.100022

13. Guo H, Duyzend MH, Coe BP, Baker C, Hoekzema K, Gerdts J, et al. Genome Sequencing Identifies Multiple Deleterious Variants in Autism Patients With More Severe Phenotypes. Genet Med (2019) 21:1611-20. doi: 10.1038/s41436018-0380-2

14. Lima Lde A, Feio-dos-Santos AC, Belangero SI, Gadelha A, Bressan RA, Salum GA, et al. An Integrative Approach to Investigate the Respective Roles of Single-Nucleotide Variants and Copy-Number Variants in Attention-
Deficit/Hyperactivity Disorder. Sci Rep (2016) 6:22851. doi: 10.1038/ srep22851

15. Cadby G, Mukherjee S, Musk AW, Reid A, Garlepp M, Dick I, et al. A Genome-Wide Association Study for Malignant Mesothelioma Risk. Lung Cancer (2013) 82:1-8. doi: 10.1016/j.lungcan.2013.04.018

16. Rokutan H, Hosoda F, Hama N, Nakamura H, Totoki Y, Furukawa E, et al. Comprehensive Mutation Profiling of Mucinous Gastric Carcinoma. J Pathol (2016) 240:137-48. doi: 10.1002/path.4761

17. Zhang Y, Mao X, Liu X, Song RR, Berney D, Lu YJ, et al. High Frequency of the SDK1:AMACR Fusion Transcript in Chinese Prostate Cancer. Int J Clin Exp Med (2015) 8:15127-36.

18. Yamagata M. Structure and Functions of Sidekicks. Front Mol Neurosci (2020) 13:139. doi: $10.3389 /$ fnmol.2020.00139

19. Mäki-Nevala S, Sarhadi VK, Knuuttila A, Scheinin I, Ellonen P, Lagström S, et al. Driver Gene and Novel Mutations in Asbestos-Exposed Lung Adenocarcinoma and Malignant Mesothelioma Detected by Exome Sequencing. Lung (2016) 194(1):125-35. doi: 10.1007/s00408-015-9814-7

20. Zhao YD, Yang J, Chen ZL, Gao ZB, Zhou F, Li XC, et al. Identification of Somatic Alterations in Stage I Lung Adenocarcinomas by Next-Generation Sequencing. Genes Chromosomes Cancer (2014) 53(4):289-98. doi: 10.1002/ gcc. 22138

21. Gristina V, La Mantia M, Iacono F, Galvano A, Russo A, Bazan V. The Emerging Therapeutic Landscape of ALK Inhibitors in Non-Small Cell Lung Cancer. Pharmaceuticals (Basel) (2020) 13(12):474. doi: 10.3390/ph13120474

22. Yang JJ, Zhou J, Yang N. SAF-189s in Previously Treated Patients With Advanced ALK-Rearranged Non-Small Cell Lung Cancer (NSCLC): Results From the Dose-Finding Portion in a Single-Arm, First-in-Human Phase I/II Study. J Clin Oncol (2020) 38:e21689-e21689. doi: 10.1200/ JCO.2020.38.15_suppl.e2168938

23. Xia ZJ, Ji YC, Sun DQ, Peng X, Gao YL, Fang YF, et al. SAF-189s, a Potent New-Generation ROS1 Inhibitor, is Active Against Crizotinib-Resistant ROS1 Mutant-Driven Tumors. Acta Pharmacol Sin (2021) 42:998-1004. doi: 10.1038/s41401-020-00513-3

24. Listì A, Barraco N, Bono M, Insalaco L, Castellana L, Cutaia S, et al. ImmunoTargeted Combinations in Oncogene-Addicted Non-Smallcell Lung Cancer. Transl Cancer Res Actions (2019) 8(Suppl 1):S55-63. doi: 10.21037/ tcr.2018.10.04

Conflict of Interest: DW and TG are employees of YuceBio Technology Co., Ltd Shenzhen.

The authors declare that the research was conducted in the absence of any commercial or financial relationships that could be construed as a potential conflict of interest.

Publisher's Note: All claims expressed in this article are solely those of the authors and do not necessarily represent those of their affiliated organizations, or those of the publisher, the editors and the reviewers. Any product that may be evaluated in this article, or claim that may be made by its manufacturer, is not guaranteed or endorsed by the publisher.

Copyright (c) $2022 \mathrm{Ma}$, Xiao, Guan, Wu, Gu and Wang. This is an open-access article distributed under the terms of the Creative Commons Attribution License (CC BY). The use, distribution or reproduction in other forums is permitted, provided the original author(s) and the copyright owner(s) are credited and that the original publication in this journal is cited, in accordance with accepted academic practice. No use, distribution or reproduction is permitted which does not comply with these terms. 